\title{
IMPLICAÇÕES DO USO DE DROGAS E A CONDIÇÃO DE SAÚDE DOS CAMINHONEIROS
}

DOI: $10.22289 / 2446-922 X . V 2 E E A 8$

\author{
Priscilla Fonseca Nascimento ${ }^{8}$ \\ Gilmar Antoniassi Junior
}

\section{RESUMO}

O uso de drogas por motoristas ocorre pelo mundo todo e as implicações decorrentes do uso são alarmantes. O objetivo do estudo foi de verificar o uso de drogas por motoristas de cargas associado à exposição do risco a condição de saúde. Trata-se de uma pesquisa quantitativa de natureza descritiva e exploratória, envolvendo os 31 motoristas, cuja foram convidados a responderem um Questionário de Exposição ao Risco, bem como questões do Teste ASSIST, considerou o IC de $95 \%$ para os testes estatísticos aplicados. Os resultados evidenciam que $25,8 \%$ já fizeram uso de drogas e a droga mais consumida são os estimulantes e $61,5 \%$ já tiveram DST. Concluiu-se que ações promotoras e preventivas da saúde devem ser implantadas dentro das empresas que possuem estes profissionais.

Palavras-chave: Uso de Drogas; Motoristas; Promoção da Saúde.

\section{ABSTRACT}

Drug use by drivers occurs around the world and the implications of the use are alarming. The aim of the study was to verify the use of drugs by freight drivers associated with exposure risk to health. This is a quantitative study of descriptive and exploratory nature, involving 31 drivers, which were invited to respond to each Risk Exposure Questionnaire, as well as issues of Test ASSIST, considered the $95 \% \mathrm{Cl}$ for the statistical tests applied. The results show that $25.8 \%$ have made use of drugs and the most consumed drugs are stimulants and $61.5 \%$ had STI. It was concluded that promoting and preventive health actions should be implemented within the companies that have these professionals.

Keywords: Drug Use; Drivers; Health promotion.

\section{INTRODUÇÃO}

Devido as grandes demandas advindas da globalização, e aos prazos curtos para entrega das mercadorias até o consumidor final, o uso de substância psicoativa, tem sido cada vez mais utilizada por motoristas profissionais 'caminhoneiros'. A maior parte das mercadorias consumida no Brasil é transportada pela malha rodoviária (1).

${ }^{8}$ Endereço eletrônico de contato: priscillabertier@hotmail.com 
O consumo do álcool, do tabaco e de outras drogas ocorre em todos os países. Mais da metade da população das Américas e da Europa já experimentou álcool alguma vez na vida. Ainda, o consumo de drogas ilícitas atinge $4,2 \%$ da população mundial, sendo a maconha a mais consumida (144 milhões de pessoas), seguida pelas anfetaminas (29 milhões), cocaína (14 milhões) e os opiláceos (13,5 milhões). As complicações clínicas e sociais causadas pelo consumo dessas substâncias, atualmente, são bem conhecidas e consideradas um problema de saúde pública que deve ser observado com bastante cautela pelos órgãos responsáveis por ações de promoção e prevenção da saúde (2).

No relatório da Organização Mundial da Saúde - OMS, feito em 2004, há indicadores de ocorrências de 1,2 milhão de mortes por acidentes de trânsito no mundo, com mais de 50 milhões de pessoas feridas que utilizaram pelo menos uma substância psicoativa. Além das mortes, existem as sequelas irreversíveis prejudicando não só o indivíduo, mas também toda a sociedade (3).

Dirigir é um exercício que requer a máxima atenção do condutor, principalmente quando este faz uso da direção como profissão. O profissional necessita estar atento a todos os estímulos externos que os torna influenciável, para guardar sua segurança e a de outras pessoas. Pois os acidentes de trânsito representam a segunda maior causa de mortalidade por causas externas e, em pessoas com idade entre 1 e 39 anos, essa estatística sobe para primeiro lugar (4).

O uso de substâncias psicoativas está cada vez mais presente no dia a dia dos motoristas 'caminhoneiros', devido os fatores de trabalho e os aspectos emocionais que este se encontra envolvido. Estes fatores incluem desde o excesso de trabalho, à pressão pela entrega no prazo mínimo estipulado, e principalmente a falta de atividades prazerosas saudáveis em torno do ambiente familiar (4).

Pesquisas apontam que o crescimento do número de condutores envolvidos em acidentes de trânsito está ligado ao ato de dirigir sob o efeito de substâncias psicoativas, principalmente o álcool, pois é sabido que os efeitos residuais aparecem mesmo quando os principais efeitos desaparecem. A ressaca do álcool afeta os reflexos e no caso das anfetaminas, o sono, causando fadiga e a degeneração neuronal que é responsável pela produção de serotonina, neurotransmissor responsável pela regulação do humor, sono, apetite, ritmo cardíaco, temperatura corporal, sensibilidade à dor, os movimentos e as funções intelectuais. Componentes indispensáveis para uma boa condução veicular (5).

Dentre os estudos publicados, as características pessoais ou alguma patologia psiquiátrica de motoristas que cometeram alguma infração ou acidente de trânsito, foram Outubro, 2016:2(Edição Especial):104-.116 
influenciados pelo uso de substância psicoativa, diante dessa informação o diagnóstico psiquiátrico ou de marcadores de risco destas morbidades são fatores determinantes para intervenções de prevenção e promoção da saúde dos motoristas caminhoneiros evitando assim possíveis recaídas ao uso e abuso de SPAS e também às infrações ou na pior das hipóteses acidentes de trânsito (6).

Assim, o objetivo deste trabalho foi de verificar o uso de drogas por motoristas de cargas associado à exposição do risco a condição de saúde. A partir da perspectiva em que se identifica os fatores que interferem na qualidade de vida no trabalho como sedentarismo, alimentação inadequada, promiscuidade associada ao uso de substâncias psicoativas.

\section{METODOLOGIA}

O estudo é do tipo quantitativo de natureza descritiva e exploratória, com 114 motoristas caminhoneiros do departamento de Logística e Transportes de uma Cooperativa de Laticínio. Os critérios de inclusão se ativeram em possuir mais de dois anos comprovados em registro de Carteira de Trabalho, e que fossem maiores de 21 anos. E foram excluídos, aqueles que não atenderam aos critérios de inclusão, que não fazem uso de rodovia estando somente no perímetro urbano do município, e que não responderam $50 \%$ dos instrumentos ou rasuraram. Optou-se por constituir a amostra por meio de levantamento que abrangesse todos os motoristas, onde a amostra foi constituída por 31 motoristas.

Utilizou-se de instrumento de coleta de dados um Questionário de Exposição ao Risco do Transito e a Qualidade de Vida de Motoristas Caminhoneiros (Apêndice - A), e o Teste para Triagem do Envolvimento com Fumo, Álcool e Outras drogas - ASSIST (Anexo A).

O questionário foi elaborado baseado nas observações da rotina diária dos motoristas de transporte de cargas, nas altas taxas de afastamentos devido condições de saúde sem a utilização de outras fontes para execução do instrumento, a fim de melhor verificar a relação do motorista com o trânsito. O Teste ASSIST é um questionário elaborado pela OMS que contém oito questões sobre o uso de nove classes de substâncias psicoativas: tabaco, álcool, maconha, cocaína, estimulantes, sedativos, inalantes, alucinógenos e opiáceos. As questões abordaram a frequência do uso na vida e nos últimos três meses, problemas relacionados ao uso, preocupação a respeito do uso por parte de pessoas 
próximas ao usuário, prejuízo na execução de tarefas esperadas, tentativas malsucedidas de cessar ou reduzir o uso, sentimento de compulsão e uso por via injetável $(7,8)$.

O processo de coleta de dados deu-se primeiramente com a sensibilização referente à proposta do estudo junto ao departamento de transporte e logística. Após o período de sensibilização, iniciou o processo de levantamento da amostra e a aplicação dos instrumentos, aos quais os motoristas caminhoneiros foram abordados de forma individual ou coletivamente, levando em consideração o momento da abordagem o convite era refeito e esclarecendo o propósito e objetivos do estudo. E com o aceite do motorista, o mesmo era convidado a primeiramente assinar o Termo de Consentimento Livre e Esclarecido (Apêndice - B) para que tomassem ciência e conhecimento da pesquisa, e todas as dúvidas esclarecidas.

A análise dos dados em relação ao questionário levou-se em conta levara em conta o agrupamento das questões definidas a fim de identificar: perfil do motorista (questão 1 a 5); condição de saúde (questão 6 a 20); comportamento de risco (questão21 a 24). E para análise dos dados do ASSIST respeitou-se a padronização do teste, considerando-se a faixa de escore de 0 a 3 como indicativa de uso ocasional, de 4 a 26 como indicativa de abuso e maior que 27 como sugestiva de dependência.

Foi realizada a análise descritiva em relação aos resultados obtidos no questionário considerou-se o agrupamento das questões definidas na estrutura de análise. A fim de, executar os cruzamentos exploratórios entre o perfil do motorista e o comportamento de exposição ao risco à saúde, verificando por meio do ASSIST o envolvimento com o uso de álcool e outras drogas. Levando em consideração $95 \%$ do nível de confiança por meio da análise bivariada, utilizando o programa Epi Info® versão 3.5.2.

A pesquisa foi aprovada pelo Comitê de Ética em Pesquisa da Universidade de Franca (UNIFRAN), sob Parecer nº 495.547 (Anexo - B).

\section{RESULTADOS}

De acordo com os dados sociodemográficos a tabela 1 permite reconhecer o perfil dos motoristas caminhoneiros correspondente a amostra do estudo. 
Tabela 1. Distribuição dos motoristas conforme dados sociodemográficos. Patos de Minas, MG, Brasil, 2013.

\begin{tabular}{|c|c|}
\hline Variáveis & $\begin{array}{l}\text { Frequência } \\
\text { [n=31 (\%)] }\end{array}$ \\
\hline \multicolumn{2}{|l|}{ Idade } \\
\hline 18 a 25 anos & $5(16,1)$ \\
\hline 26 a 35 anos & $13(42)$ \\
\hline 36 a 45 anos & $8(25,8)$ \\
\hline 46 a 50 anos & $2(6,5)$ \\
\hline Acima de 51 anos & $3(9,7)$ \\
\hline \multicolumn{2}{|l|}{ Estado Civil } \\
\hline Casado & $16(51,6)$ \\
\hline União Estável & $3(9,7)$ \\
\hline Solteiro & $11(35,5)$ \\
\hline Divorciado & $1(3,2)$ \\
\hline Viúvo & - \\
\hline \multicolumn{2}{|l|}{ Escolaridade } \\
\hline Ensino fundamental completo & $3(9,7)$ \\
\hline Ensino fundamental incompleto & $5(16,1)$ \\
\hline Ensino médio completo & $14(45,2)$ \\
\hline Ensino médio incompleto & $8(25,8)$ \\
\hline Superior incompleto & $1(3,2)$ \\
\hline \multicolumn{2}{|l|}{ Filhos } \\
\hline Sim & $1(33,3)$ \\
\hline Não & $2(66,7)$ \\
\hline
\end{tabular}

No que refere ao uso do tabaco $48,4 \%$ nunca fizeram uso do tabaco e $51,6 \%$ já fizeram uso do tabaco. Dos que já fizeram uso do cigarro, $75 \%$ são fumantes e $25 \%$ não. Sendo que dos fumantes $50 \%$ fazem uso acima de 20 cigarros por dia, seguido de $33,3 \%$ entre 15 a 20 cigarros. Quanto ao uso do álcool 64,5\% fazem uso e 35,5\% não fazem. A bebida mais consumida pelos motoristas quando estão viajando, na amostra de usuários é a água $85 \%$, seguido da cerveja $40 \%$ e do refrigerante $40 \%$. Entre os não usuários a água $74,8 \%$ é a mais consumida, seguido do refrigerante $45,5 \%$ e $27,3 \%$ de suco natural. A tabela 2 permite compreender os dados referentes ao uso de outras drogas que não álcool e tabaco na vida dos motoristas por meio do ASSIST.

Outubro, 2016:2(Edição Especial):104-.116 
Tabela 2. Distribuição dos motoristas conforme o uso de outras drogas na vida. Patos de Minas, MG, Brasil, 2013.

\begin{tabular}{|c|c|}
\hline Substância & $\begin{array}{c}\text { Frequência } \\
{[n=8(\%)]}\end{array}$ \\
\hline Maconha & $6(75)$ \\
\hline Cocaína/ Crack & $5(62,5)$ \\
\hline Estimulantes & $7(87,5)$ \\
\hline Inalantes & $2(25)$ \\
\hline Hipnóticos & $3(37,5)$ \\
\hline Drogas Alucinógenas & $2(25)$ \\
\hline Opióides & $2(25)$ \\
\hline Outras & - \\
\hline
\end{tabular}

Ao verificar a associação do cansaço com o uso de cigarro dos que fazem uso do cigarro, evidenciou que somente $41,7 \%$ dos motoristas nunca associaram o cansaço com o uso do cigarro, 33\% raramente associaram o cansaço ao uso do cigarro, $16,7 \%$ às vezes faz esta associação e $8,3 \%$ relaciona o cansaço ao uso do cigarro na maioria das vezes. No que refere ao uso do álcool e outras drogas, quanto à dificuldade de concentração, 66,7\% dos que fazem uso somente do álcool raramente sentem dificuldades e dos que associam álcool e outra droga $75 \%$ também raramente apresenta dificuldade, no entanto $12,5 \%$ dos que associam na maioria das vezes percebem tais dificuldades.

Com relação à qualidade do sono dos motoristas pode evidenciar quanto à dificuldade para dormir após longas horas de estradas, que 61,3\% nunca sentiram dificuldades para dormir, 22,6\% às vezes sentiram alguma dificuldade, 12,9\% raramente demonstraram dificuldades para dormir e 3,2\% na maioria das vezes demonstrou dificuldades para dormir após longas horas de estrada. E avalia a qualidade do sono como bom $32,3 \%$ da amostra, sendo que $32,3 \%$ avaliam com sendo muito bom, $32,3 \%$ avalia como nem bom nem ruim e $3,2 \%$ avalia a qualidade do sono com ruim. E quando apresenta algum problema relativo ao sono $29 \%$ não se preocupa nada, $25,8 \%$ se preocupa bastante, $22,6 \%$ se preocupa muito pouco, $16,1 \%$ se preocupa mais ou menos e $6,5 \%$ ficam extremamente preocupados com algum problema relativo ao sono. Com relação a problemas relacionados à saúde, a tabela 3 permite identificar a condição dos motoristas a partir de 5 afirmativas expressas na amostra.

Outubro, 2016:2(Edição Especial):104-.116 
Tabela 3. Distribuição dos motoristas em relação a condição de saúde. Patos de Minas, MG, Brasil, 2013.

\begin{tabular}{lcccc}
\hline \multicolumn{1}{c}{ Variáveis ${ }^{(\mathrm{n}=31)}$} & Sim & Não & Não Sabe \\
\hline $\begin{array}{l}\text { Possui algum tipo de doença } \\
\text { trabalho. }\end{array}$ & $5(16,1)$ & $23(74,2)$ & $3(9,7)$ \\
$\begin{array}{l}\text { Sofre de problemas de hipertensão. } \\
\text { Sofre de diabetes. }\end{array}$ & $4(12,9)$ & $22(71)$ & $5(16,1)$ \\
Sentiu alguma dificuldade respiratória. & $8(25,8)$ & $16(51,6)$ & $7(22,6)$ \\
Alguma vez na vida já teve DST & $13(41,9)$ & $15(48,4)$ & $3(9,7)$ \\
\hline
\end{tabular}

Associando o consumo do álcool e os problemas com doenças sexualmente transmissíveis - DST, dentre a amostra de usuários de álcool $50 \%$ já tiveram alguma DST, $45 \%$ não sabem se tiveram e 5\% nunca tiveram nenhuma doença. Dos 41,9\% que indicaram ter tido alguma DST, ao qual representa $60 \%$ que são usuários de álcool e $40 \%$ que são usuários de droga, indicando $12 \%$ de possibilidade que o evento venha ocorrer entre os usuários de álcool e droga que possa ter tido alguma DST. Destes que já tiveram uma DST na amostra geral apenas $61,5 \%$ fizeram tratamento de saúde, e 38,5\% não fizeram tratamento de saúde. Ao qual verifica que na amostra de usuários de álcool que já tiveram alguma DST, $60 \%$ fizeram tratamento e $40 \%$ não fizeram tratamento, o que representa $68 \%$ de possibilidades de se repetir, e na amostra de usuários de drogas $66,7 \%$ fizeram tratamento e $33,3 \%$ não fizeram tratamento, o que representa $56 \%$ de possibilidade de se repetir na amostra de usuários de droga.

\section{DISCUSSÃO}

Na amostra identificou-se que $100 \%$ dos envolvidos são do sexo masculino, com idade entre 26 a 35 anos, com nível de escolaridade médio completo, onde a maioria é casado, e não possuem filhos. Dados este são semelhantes ao estudo sobre o estilo de vida de motoristas de caminhão que, evidenciou $100 \%$ são do sexo masculino, a maioria tinha mais de 30 anos (75\%), eram casados e com filhos (80\%) e $64 \%$ de baixa escolaridade (9).

Estudo aponta que $21 \%$ dos motoristas caminhoneiros fazem uso do cigarro e consomem em média 17,1 cigarros por dia. O uso do cigarro pode ser compreendido devido o fator estressante de horas e horas no volante. Porém é válido ressaltar que o número de usuários de cigarros tem caído devido às ações de prevenção ao uso e promoção a saúde, 
por meio de campanhas efetivas de entidades como INCA. Através da aliança por um mundo sem tabaco cujo objetivo é de acompanhar e participar dos processos de controle do tabagismo no Brasil e no mundo, discutindo temas transversais em saúde, educação, direito das crianças e mulheres, legislação, meio ambiente e economia e a pressionarem os legisladores a tomarem as providências necessárias para termos um mundo sem tabaco $(9,10)$.

É evidente que o uso do álcool entre motoristas é um tema preocupante no contexto atual, devido às graves consequências e aos altos índices de acidentes de trânsito que tem gerado. Dados estes que se compactuam com o levantamento realizado em 2004 no Brasil, ao qual indicam que os acidentes de trânsito foram responsáveis pela perda de 35.674 vidas, sendo a nona causa principal de morte e a segunda entre as causas externas, sendo que os homicídios aparecem em primeiro lugar (4).

Em alusão ao uso de outras drogas que não ao álcool e tabaco, estudos apontam que $70 \%$ dos caminhoneiros fazem uso de drogas para manterem-se acordados, este é o discurso de muitos, mas é sabido que existem outros fatores que possam influenciar o abuso destas substâncias (10). Evidencia-se que o uso de estimulantes, é devido alegações dos motoristas para que possam se manter acordados por longos períodos de tempo. $\mathrm{O}$ alto uso de anfetaminas entre caminhoneiros é indicado em estudos com os caminhoneiros brasileiros (11).

No estudo realizado com motoristas de caminhão nas rodovias paulistas, ao qual testara amostra de urina indicaram que $5,8 \%$ dos motoristas fazem uso. O que se confirma no estudo realizado com caminhoneiros da região Sudeste, Nordeste e Sul do Brasil, indicando que $4,8 \%$ fazem uso, destacando a região sul $6 \%$ como região de maior uso e a região nordeste $3,7 \%$ de uso $(12,13)$.

O fato de que os motoristas de caminhão estão sob a pressão do tempo devido a entrega restrita, e viver frente à exposição e ao risco, e com o tempo restrito ao lazer, pode evidenciar a fuga da constante pressão ao uso de drogas (9).

Entretanto, o estudo aponta a maconha, cocaína e o crack como substâncias recorrentes de uso entre motoristas, evidenciando que somente o fato de se usar os estimulantes os mesmos não estão sendo capazes de satisfazer e tencionando ao uso das demais drogas, a fim de direcionar para alternativas diferentes de satisfação. Em concordância com os achados, estudo realizado pela Polícia Rodoviária Federal, em 2010, através de exames toxicológicos com caminhoneiros voluntários do Espírito Santo, revelou que um em cada três motoristas dirigia sob o efeito de alguma droga. O mesmo estudo revela Outubro, 2016:2(Edição Especial):104-.116 
que $30 \%$ dos caminhoneiros fazem o uso frequente de droga. Relato dos motoristas apontam que a utilização das drogas é para mantê-los acordados e conseguirem trabalhar mais horas seguidas (14).

Porém o efeito do uso das drogas leva a inúmeros problemas de saúde tais como: inapetência, insônia, midríase, agressividade, taquicardia, intoxicações, tremores, sudorese, alucinações e a morte, onde estudos semelhantes apontam que $20 \%$ dos sujeitos que usam droga relataram apresentar diversos destes efeitos $(10,15,16)$.

O ato de dirigir sob o efeito de álcool e outras drogas configura-se em comportamento de exposição ao risco, o que aumenta a possibilidade de se envolver em acidentes ou demais problemas. O que torna importante se ter uma percepção clara da decisão a tomar, além dos riscos a serem assumidos, pois é preciso ter a compreensão do limite de convivência a exposição ao risco $(15,16,17,18)$.

O estudo possibilita perceber que a condição de saúde em relação a qualidade de vida, tem se desvalido em segundo plano, pois grande parte dos participantes, isto nos permitiu verificar que alguns profissionais mesmo que poucos se preocupam com a saúde e que consideram como uma prioridade para melhorar a qualidade de vida e se envolvem com os cuidados de saúde o que raramente são fatores preocupantes como demonstra a pesquisa, pois eles quase não se cansam com facilidade e não possuem alguma dificuldade de concentração ou desconforto causado pelo desempenho de suas funções. Dados estes que se confirmam no estudo com motoristas caminhoneiros em Campinas, onde grande parte dos participantes da pesquisa referiu não ir ao médico há muito tempo, não utilizavam os serviços de saúde quando estão viajando, às vezes apenas verificam a pressão arterial, e dizem não terem tempo para cuidar da saúde $(14,15,16)$.

Estudos com motoristas de caminhão, no Brasil, Ásia, África, alertam para importantes problemas com relação a esta atividade profissional. Os dados expõem que os problemas relacionados às condições de saúde, trabalho e estilo de vida, se fazem vulneráveis a exposição às práticas de risco, acrescido pelo uso das drogas e pela diversidade nas parceiras sexuais eventuais. Comportamentos que alertam as condições de trabalho e do estilo de vida, e que afetam diretamente a saúde chamando a atenção da psicologia em termos de saúde do trabalhador, referenciando as ações promotoras à saúde $(17,18,19)$.

Verificado a exposição ao risco da saúde sexual, o estudo possibilitou evidenciar que alguma vez na vida tiveram alguma DST. O que pode evidenciar a possibilidade de que a contaminação com alguma DST está mais suscetível pelas alterações psíquicas provocadas pelo uso do álcool e/ou outras drogas. Estudos realizados no Brasil têm demonstrado a Outubro, 2016:2(Edição Especial):104-.116 
vulnerabilidade dos caminhoneiros em relação à AIDS, dessa forma, diversas estratégias de intervenção precisam ampliar o envolvimento destes trabalhadores, em processos que os tornem em mecanismos mais eficazes de educação sobre os riscos de contaminação pelo HIV, pela alienação a preservação. A falta de prevenção dos caminhoneiros demonstrou mais influencias das ideias simplistas e ingênuas em torno das DST's e AIDS. A maioria refere não fazer uso de preservativo (20).

Os aspectos culturais são próprios do gênero masculino, que tornam os homens mais vulneráveis às práticas de risco para as DST/AIDS, tais como: sentir-se forte, imune a doenças; ser impetuoso, correr riscos; ser incapaz de recusar uma mulher; considerar que o homem tem mais necessidade de sexo do que a mulher e de que esse desejo é incontrolável. A infidelidade masculina é considerada natural; a feminina é atribuída a deficiências do parceiro $(15,21)$.

Estes dados refletem a necessidade do desenvolvimento ações programadas que visem à educação e as intervenções possam estar voltadas a prevenção de DST/AIDS e a promoção da saúde do caminhoneiro em ações conjuntas de participativa que refletem em mudanças de estilo de vida e investimento na qualidade de vida. Segundo o Ministério da Saúde a campanha de prevenção às DST's/ AIDS neste ano de 2014 será estendida a todos os grandes eventos e festas populares, que possa mobilizar a sociedade, pretendendo alertar o risco e a necessidade da prevenção nos momentos de divertimento e prazer. Alertando sobre a importância da testagem (22).

\section{CONCLUSÃO}

Diante da proposta do estudo, foi possível verificar um número expressivo de motoristas que fazem uso de drogas, principalmente o álcool e a cocaína. É válido ressaltar que mesmo após sansão da lei seca artigo 306 do Código Brasileiro de Trânsito - alterada pela lei 12760/12, o estudo aponta que os motoristas não se intimidam em associar o uso das substâncias psicoativas com a direção. Com isso, percebe-se que o uso indiscriminado de drogas envolvendo motoristas está cada vez mais presente nas rodovias, o que tem contribuído com a degradação social e pessoal do sujeito.

O fato é que, o uso destas substâncias leva o sujeito à exposição do risco vulnerável à saúde, a se tornarem dependentes dessas substâncias, conforme resultados do ASSIST revela que pouco mais de $50 \%$ utilizam pelo menos alguma vez ao dia alguma substância psicoativa aumentando assim a probabilidade do vício. É salutar o diálogo com as categorias Outubro, 2016:2(Edição Especial):104-.116 
de motoristas, para que de fato a condição de exposição ao risco da saúde, possa ser percebida efetivamente pelo sujeito envolvido a fim de provocar a mudança de atitude necessária à qualidade de vida.

No que refere ao comportamento de risco relativo às relações sexuais, os resultados apontam para com o comportamento negligenciado em relação as doenças sexualmente transmissíveis e a AIDS. Evidenciando um problema para a saúde pública, relacionado aos agravos que estas doenças trazem. É importante lembrar que os homens estão mais expostos aos riscos da promiscuidade, pois iniciam sua vida sexual mais cedo, o que faz com tenham uma variedade parceiros sexuais, o que aumenta a probabilidade do risco a saúde sexual, quando não prevenida. A masculinidade encontra-se em jogo, nas relações sexuais.

Por fim, o estudo possibilita sugerir que sejam implantados dentro das empresas grupos terapêuticos com estes profissionais, estando focados em ações de prevenção e promoção da saúde do trabalhador, levando informações sobre os prejuízos pessoais, sociais e cognitivos que o uso destas substâncias pode ocasionar, implantando rodas de conversa, terapia breve nos que já fazem uso ocasional.

\section{REFERÊNCIAS}

1. Centro Brasileiro de Informações Sobre Drogas Psicotrópicas/Anfetaminas. [acesso em 1 nov 2013]. Disponível em: http://www.unifesp.br/dpsicobio/cebrid/folhetos/anfetaminas.

2. Marangon SR, Oliveira MLF. Uso de crack por multípara em vulnerabilidade social: história de vida. Rev Cienc Cuid Saude. 2012;11(1):166-172.

3. Barczakl R, Duarte F. Impactos ambientais da mobilidade urbana: cinco categorias de medidas mitigadoras. Rev. Bras. Gest. Urbana. 2012; 4(1):13-32.

4. Ponce JC, Leyton V, Drogas ilícitas e trânsito: problema pouco discutido no Brasil. Rev Psiq Clínica. 2008; 35(Suppl 1):65-69.

5. Takitane J, Oliveira LG, Endo LG, Oliveira KCBG, Muñoz DR, Yonamine M et al . Uso de anfetaminas por motoristas de caminhão em rodovias do Estado de São Paulo: um risco à ocorrência de acidentes de trânsito?. Ciênc Saúde Coletiva. 2013;18(5):1247-1254. 
6. McMillan G, et al. Underdiagnosis of comorbid mental illness in repeat DUI offenders mandated to treatment. J Subst Abuse Treat. 2008;34(3):320-325.

7. Who. Assist Working Group. The alcohol, Smoking and substance Involvement Screening Test (ASSIST): development, reliability and feasibility. Addiction 2002; (97):1183-1194.

8. Henrique IFS, De MD, Lacerda RB, Lacerda LA, Formigoni MLOS. Validação da versão brasileira do teste de triagem do envolvimento com álcool, cigarro e outras substâncias (ASSIST). Rev Assoc Med Bras. 2004;50(2):199-206.

9. Masson VA, Monteiro MI. Vulnerabilidade à Doenças Sexualmente Transmissíveis/AIDS e uso de drogas psicoativas por caminhoneiros. Rev Bras Enferm 2010;63(1):79-83.

10. Saito T, Yamamoto I, Hhuang X. Determination of chronic methamphetamine abuse by hair analysis. Forensic Sci Int. 2000;112(1):65-71.

11. Nappo SA, Carlini EA, Araújo MD, Moreira LFS. Prescription of anorectic and benzodiazepine drugs through notification B prescriptions in Natal, Rio Grande do Norte, Brazil. Braz J Pharm Sci 2010; 46(2):297-303.

12. Alessi A, Alves MK. Hábitos de vida e condições de saúde dos caminhoneiros do Brasil: uma revisão da literatura. Rev Ciência e Saúde. 2015;8(3):129-136.

13. Souza JC, Paiva T, Reimão R. Sono, qualidade de vida e acidentes em caminhoneiros brasileiros e portugueses. Psicol Estud. 2008; 13(3):429-36.

14. Silva OA, Greve JMD, Yonamine M, Leyton V. Drug use by truck drivers in Brazil. Drugs Educ Prev Policy. 2003;10(2):135-9.

15. Brandão FR; Antoniassi Junior G. O uso de substâncias nocivas associadas ao comportamento de risco do praticante de atividade física. Psicol e Saúde em Debate;20151(1):53-64.

16. Domingos JBC, Jora NP, Carvalho AMP, Pillon SC. Consumo de álcool, sobrepeso e obesidade entre caminhoneiros. Rev Enferm UERJ. 2010;18(3):377-82.

17. Musshoff F, Madea B. Driving under the influence of amphetamine-like drugs. J Forensic Sci. 2012;57(2):413-419.

18. Medeiros LKA. As marcas dos Hospitais Psiquiátricos: um relato de experiência. Psicol e Saúde e Debate. 2015;1(2):20-34. 
19. Guedes HM, Brum KA, Costa PA, Almeida MEF. Fatores de Risco para o Desenvolvimento de de Hipertensão Arterial entre Motoristas Caminhoneiros. Cogitare Enferm. 2010;15(4):652-658.

20. Leyton V, Sinagawa DM, Oliveira KCBG, Schmitz W, Andreuccetti G, De Martinis BS, Yonamine M, Muñoz DR. Amphetamine, cocaine and cannabinoids use among truck drivers on the roads in the State of Sao Paulo, Brazil. Forensic Sci Int. 2012;215(1-3):25-27.

21. Murphy DA, Hser YI, Huang D, Brecht ML, Herbeck DM. Self-report of Longitudinal Substance Use: A Comparison of the UCLA Natural History Interview and the Addiction Severity Index. J Drug Issues. 2010; 40(2):495-516.

22. Balbinot AB, Timm MI, Zaro MA. Jogo TransRisco: identificação do comportamento de risco em condutores. Rev Novas Tecnol na Educação. 2010;8(3):1-11. 